\title{
Explicit upper bounds for $|L(1, \chi)|$ for primitive even Dirichlet characters
}

by

\section{Stéphane Louboutin (Marseille)}

1. Introduction. Let $p_{1}, \ldots, p_{r}$ be $r \geq 0$ given positive distinct rational primes and let $\chi$ range over the primitive (even or odd) Dirichlet characters of conductors $f_{\chi}>1$. In [Lou1] we explained how to compute a reasonable constant $\kappa_{r}$, depending on the $p_{i}$ 's only, such that

$$
\left|\prod_{i=1}^{r} 1-\frac{\chi\left(p_{i}\right)}{p_{i}}\right||L(1, \chi)| \leq \frac{1}{2}\left(\prod_{i=1}^{r}\left(1-\frac{1}{p_{i}}\right)\right) \log f_{\chi}+\kappa_{r} .
$$

More explicitly, a careful analysis of our proof shows that we may take

$$
\kappa_{r}=R_{r}(1)+R_{r}(\chi)+\left|\prod_{i=1}^{r} 1-\frac{\chi\left(p_{i}\right)}{p_{i}}\right|
$$

where $R_{r}(1)$ and $R_{r}(\chi)$ are computed inductively from the formulae

$$
\begin{aligned}
& R_{0}(1)=R_{0}(\chi)=0, \\
& R_{r+1}(1)=\frac{\varepsilon_{\chi}+\log p_{r+1}}{p_{r+1}}+\left(1-\frac{1}{p_{r+1}}\right) R_{r}(1), \\
& R_{r+1}(\chi)=\left|\chi\left(p_{r+1}\right)\right| \frac{\varepsilon_{\chi}+\log p_{r+1}}{p_{r+1}}+\left|1-\frac{\chi\left(p_{r+1}\right)}{p_{r+1}}\right| R_{r}(\chi),
\end{aligned}
$$

where $\varepsilon_{\chi}=(\chi(1)-\chi(-1)) / 2 \in\{0,1\}$. In particular, $R_{r}(\chi)=0$ if $\chi\left(p_{i}\right)=0$ for all the $r$ primes $p_{i}$. Moreover, $R_{r}(1) \leq \sum_{i=1}^{r}\left(\varepsilon_{\chi}+\log p_{r}\right) / p_{r}$. For a given set of $r$ primes $p_{i}$, one needs a little time to determine which ordering of the $p_{i}$ 's will yield the least values for $R_{r}(1)$ and $R_{r}(\chi)$. For example, if we let $\chi$ range over the primitive even Dirichlet characters such that $\chi(2)=0$ and $\chi(3)=-1$, then the choice $p_{1}=2$ and $p_{2}=3$ yields $R_{2}(1)=\frac{1}{6} \log (36)$ and $R_{2}(\chi)=\frac{1}{3} \log 3$, whereas the choice $p_{1}=3$ and $p_{2}=2$ yields $R_{2}(1)=$ $\frac{1}{6} \log (24)$ and $R_{2}(\chi)=\frac{1}{3} \log 3$. Therefore, we obtain

(1) $|L(1, \chi)| \leq \frac{1}{8}\left(\log f_{\chi}+\kappa\right) \quad$ with $\kappa=\log (216)+8=13.375278 \ldots$

2000 Mathematics Subject Classification: Primary 11M20; Secondary 11R42, 11R29.

Key words and phrases: $L$-functions, Dirichlet characters. 
for any primitive even Dirichlet character of conductor $f_{\chi}$ satisfying $\chi(2)=0$ and $\chi(3)=-1$. This is the bound quoted in [Le, Lemma 3].

The aim of this paper is to provide the reader with better values for these constants $\kappa_{r}$ for even characters. It must be pointed out that neither of the two methods we develop here for improving upon these constants $\kappa_{r}$ 's apply to odd characters (see [Lou3] and [Ram] for explicit bounds for $|L(1, \chi)|$ for odd primitive characters).

2. Statement of the results. We let $\gamma=0.5772156649 \ldots$ denote Euler's constant.

2.1. Bounds in the case that $\chi\left(p_{i}\right)=0$ for all $i$ 's

Theorem 1. Set $\kappa_{0}=2+\gamma-\log (4 \pi)=0.046 \ldots$ Fix $k=\prod_{i=1}^{r} p_{i} \geq 1$, a square-free integer, and let $\chi$ range over the primitive even Dirichlet characters $\chi$ whose conductors $f_{\chi}>1$ are divisible by $k$. We have

$$
|L(1, \chi)| \leq \frac{1}{2}\left\{\prod_{i=1}^{r}\left(1-\frac{1}{p_{i}}\right)\right\}\left(\log f_{\chi}+\kappa_{0}+2 \sum_{i=1}^{r} \frac{\log p_{i}}{p_{i}-1}\right)+o(1)
$$

where o(1) is an explicit error term which tends rapidly to zero when $f_{\chi}$ goes to infinity. Moreover,

1. For any primitive even Dirichlet character of conductor $f_{\chi}>1$ we have

$$
|L(1, \chi)| \leq \frac{1}{2}\left(\log f_{\chi}+\kappa_{0}\right) .
$$

2. For any given prime $p \geq 2$ and for any primitive even Dirichlet character of conductor $f_{\chi} \geq f_{p}:=\frac{3}{2 \pi^{2}} p^{2}$ divisible by $p$ we have

$$
|L(1, \chi)| \leq \frac{1}{2}\left(1-\frac{1}{p}\right)\left(\log f_{\chi}+\kappa_{0}+2 \frac{\log p}{p-1}\right) .
$$

3. For any given primes $p>q \geq 2$ and for any primitive even Dirichlet character of conductor $f_{\chi} \geq f_{p, q}:=\frac{\log 4}{3 \pi} p^{2} q^{2}$ divisible by $p$ and $q$ we have

$$
|L(1, \chi)| \leq \frac{1}{2}\left(1-\frac{1}{p}\right)\left(1-\frac{1}{q}\right)\left(\log f_{\chi}+\kappa_{0}+2 \frac{\log p}{p-1}+2 \frac{\log q}{q-1}\right) .
$$

COROllary 2. Let $\chi$ be a primitive even Dirichlet character of conductor $f_{\chi}>1$. We have

$$
|L(1, \chi)| \leq A_{\chi}\left(\log f_{\chi}+\kappa_{\chi}\right)
$$

where $A_{\chi}$ and $\kappa_{\chi}$ which depend only on the value of $f_{\chi}$ modulo 6 are given in the following table: 


\begin{tabular}{cccl}
\hline $2 \mid f_{\chi} ?$ & $3 \mid f_{\chi} ?$ & $A_{\chi}$ & \multicolumn{1}{c}{$\kappa_{\chi}$} \\
\hline No & No & $1 / 2$ & $2+\gamma-\log (4 \pi)=0.046191 \ldots$ \\
No & $Y e s$ & $1 / 3$ & $2+\gamma-\log (4 \pi / 3)=1.144803 \ldots$ \\
Yes & No & $1 / 4$ & $2+\gamma-\log \pi=1.432485 \ldots$ \\
Yes & Yes & $1 / 6$ & $2+\gamma-\log (\pi / 3)=2.531098 \ldots$ \\
\hline
\end{tabular}

Proof. $f_{2}=0.6 \ldots, f_{3}=1.36 \ldots$ and $f_{2,3}=5.29 \ldots$

REMARK 3. Let us point out that lately Ramaré has been able to improve slightly upon two of these bounds (see [Ram]):

\begin{tabular}{cccc}
\hline $2 \mid f_{\chi} ?$ & $A_{\chi}$ & $\kappa \chi$ & $\kappa_{\text {Ramaré }}$ \\
\hline No & $1 / 2$ & $2+\gamma-\log (4 \pi)=0.046191 \ldots$ & 0 \\
Yes & $1 / 4$ & $2+\gamma-\log \pi=1.432485 \ldots$ & $\log 4=1.38629 \ldots$ \\
\hline
\end{tabular}

See also [Lou5] for slightly worse improvements.

2.2. Bounds in the case that $\chi\left(p_{i}\right) \neq 0$ for some $i$

Theorem 4. Set $\kappa_{0}^{\prime}=2 \gamma-1=0.154 \ldots$ Fix $h=\prod_{i=1}^{s} p_{i} \geq 1$ and $k=\prod_{i=s+1}^{r} p_{i} \geq 1$, two coprime square-free integers. Set

$$
\kappa=s \log 2-\sum_{i=1}^{s} \log \left(1-\frac{1}{p_{i}}\right)+\sum_{i=1}^{r} \frac{\log p_{i}}{p_{i}-1}
$$

and let $\chi$ range over the primitive even Dirichlet characters of conductors $f_{\chi}>1$ such that $k$ divides $f_{\chi}$. Then

$$
\left|\prod_{i=1}^{s}\left(1-\frac{\chi\left(p_{i}\right)}{p_{i}}\right)\right||L(1, \chi)| \leq \frac{1}{2}\left\{\prod_{i=1}^{r}\left(1-\frac{1}{p_{i}}\right)\right\}\left(\log f_{\chi}+\kappa_{0}^{\prime}+2 \kappa\right)+o(1)
$$

where $o(1)$ is an error term which approaches zero as $f_{\chi}$ goes to infinity.

Since $\kappa_{0}^{\prime}=2 \gamma-1=0.154 \ldots>0.046 \ldots=\kappa_{0}=2+\gamma-\log (4 \pi)$, Theorem 1 provides us with slightly better bounds than Theorem 4 for $s=0, r \geq 0$. Moreover, the error term $o(1)$ in (2) is much smaller and easier to handle than the one in (7). The error term $o(1)$ in Theorem 4 is explicit and depends on the $p_{i}$ 's. For example, we will prove:

Theorem 5. 1. Set $\kappa^{\prime}=2 \gamma+6 \log 2-1=4.313314 \ldots$ and let $\chi$ range over the primitive even Dirichlet characters of conductors $f_{\chi}>1$. Then

$$
\left|\left(1-\frac{\chi(2)}{2}\right) L(1, \chi)\right| \leq \frac{1}{4}\left(\log f_{\chi}+\kappa^{\prime}\right)+o(1)
$$

and

$$
\left|\left(1-\frac{\chi(2)}{2}\right) L(1, \chi)\right| \leq \frac{1}{4}\left(\log f_{\chi}+5\right) .
$$


In particular, if $\chi(2)=-1$ then

$$
|L(1, \chi)| \leq\left(\log f_{\chi}+5\right) / 6 .
$$

2. Set $\kappa^{\prime \prime}=\log (108)+2 \gamma-1=4.836656 \ldots$ and let $\chi$ range over the primitive even Dirichlet characters of even conductors $f_{\chi}>1$. Then

$$
\left|\left(1-\frac{\chi(3)}{3}\right) L(1, \chi)\right| \leq \frac{1}{6}\left(\log f_{\chi}+\kappa^{\prime \prime}\right)+o(1)
$$

and

$$
\left|\left(1-\frac{\chi(3)}{3}\right) L(1, \chi)\right| \leq \frac{1}{6}\left(\log f_{\chi}+6\right) .
$$

In particular, if $\chi(2)=0$ and $\chi(3)=-1$ then

$$
|L(1, \chi)| \leq\left(\log f_{\chi}+6\right) / 8 .
$$

Notice that (11) is better than (1) used in [Le, Lemma 3]. We also refer the reader to [Pin] and [Toy] for asymptotic bounds for $|L(1, \chi)|$. These bounds are asymptotically better than our bounds (2), (3) and (7), but they are not explicit and are of no practical use when applying bounds for $|L(1, \chi)|$ to class number problems for CM-fields, as in the last section of the present paper.

Corollary 6. Let $\mathbf{N}$ be a real abelian number field of degree $n>1$. Let

$$
E_{\mathbf{N}}(p)=\prod_{\mathcal{P} \mid p}\left(1-N(\mathcal{P})^{-1}\right)^{-1}
$$

denote the Euler factor of the Dedekind zeta function $\zeta_{\mathbf{N}}$ of $\mathbf{N}$ related to the rational prime $p \geq 2$. Then

$$
\operatorname{Res}_{s=1}\left(\zeta_{\mathbf{N}}\right) \leq \frac{1}{2^{n}} E_{\mathbf{N}}(2)\left(\frac{1}{2(n-1)} \log d_{\mathbf{N}}+2.5\right)^{n-1} .
$$

Proof. Let $X_{\mathbf{N}}$ be the group of order $n$ of primitive even characters associated with $\mathbf{N}$. Then

$$
\operatorname{Res}_{s=1}\left(\zeta_{\mathbf{N}}\right)=\prod_{\chi \in X_{\mathbf{N}} \backslash\{1\}} L(1, \chi), \quad d_{\mathbf{N}}=\prod_{\chi \in X_{\mathbf{N}} \backslash\{1\}} f_{\chi}
$$

and

$$
E_{\mathbf{N}}(p)=\prod_{\chi \in X_{\mathbf{N}}}\left(1-\chi(p) p^{-1}\right)^{-1}=2 / \prod_{\chi \in X_{\mathbf{N}} \backslash\{1\}}\left(1-\chi(p) p^{-1}\right) .
$$

Since the geometric mean is less than or equal to the arithmetic mean, using (8) we obtain the desired result. 
Since $E_{\mathbf{N}}(2) \leq 2^{n},(12)$ improves upon the bound

$$
\operatorname{Res}_{s=1}\left(\zeta_{\mathbf{N}}\right) \leq\left(\frac{1}{2(n-1)} \log d_{\mathbf{N}}+\frac{1}{2} \kappa_{0}\right)^{n-1}
$$

we would obtain by using (2) (instead of (8)). For example, if 2 is inert in $\mathbf{N}$ then $E_{\mathbf{N}}(2)=\left(1-2^{-n}\right)^{-1}$ and (12) is then a $\left(2^{n}-1\right)$-fold improvement on (13).

3. Proof of Theorem 1. Our proof is based on the method introduced in [Lou2]. Let $x>0$. We set

$$
g(x)=\sum_{n \geq 1} e^{-\pi n^{2} x}
$$

which satisfies the functional equation

$$
g(1 / x)=\sqrt{x} g(x)+(\sqrt{x}-1) / 2,
$$

from which we obtain the integral representation

$$
\pi^{-s / 2} \Gamma\left(\frac{s}{2}\right) \zeta(s)=\int_{0}^{\infty} x^{s / 2} g(x) \frac{d x}{x}=\int_{1}^{\infty} g(x) x^{s / 2} \frac{d x}{x}+\int_{1}^{\infty} g\left(\frac{1}{x}\right) x^{-s / 2} \frac{d x}{x}
$$

and

$$
\pi^{-s / 2} \Gamma\left(\frac{s}{2}\right) \zeta(s)=\frac{1}{s}-\frac{1}{s-1}+\int_{1}^{\infty}\left(x^{s / 2}+x^{(1-s) / 2}\right) g(x) \frac{d x}{x} .
$$

In the same way, set $f=f_{\chi}$ and

$$
g(x, \chi)=\sum_{n \geq 1} \chi(n) e^{-\pi n^{2} x / f} \quad(x>0),
$$

which satisfies the functional equation $g(1 / x, \chi)=W_{\chi} \sqrt{x} g(x, \bar{\chi})$ where

$$
W_{\chi}=\frac{1}{\sqrt{f}} \sum_{a=0}^{f-1} \chi(a) e^{2 a \pi i / f}
$$

We obtain

$$
\left(\frac{f}{\pi}\right)^{s / 2} \Gamma\left(\frac{s}{2}\right) L(s, \chi)=\int_{1}^{\infty}\left(g(x, \chi) x^{s / 2}+W_{\chi} g(x, \bar{\chi}) x^{(1-s) / 2}\right) \frac{d x}{x}
$$

Noticing that $\left|W_{\chi}\right|=1$, the choice $s=1$ yields

$$
\sqrt{f}|L(1, \chi)| \leq \int_{1}^{\infty}|g(x, \chi)| \frac{d x}{\sqrt{x}}+\int_{1}^{\infty}|g(x, \bar{\chi})| \frac{d x}{x} .
$$

Since $\chi(n)=0$ whenever $\operatorname{gcd}(n, f)>1$, we have

$$
|g(x, \bar{\chi})|=|g(x, \chi)| \leq \sum_{\substack{n \geq 1 \\ \operatorname{gcd}(n, k)=1}} e^{-\pi n^{2} x / f}=\sum_{d \mid k} \mu(d) g\left(d^{2} x / f\right),
$$


where $\mu$ denotes the Möbius function. Set

$$
I(X)=\int_{1}^{\infty}(\sqrt{x}+1) g(x / X) \frac{d x}{x} .
$$

Using (18) and (19), we obtain

$$
\sqrt{f}|L(1, \chi)| \leq \sum_{d \mid k} \mu(d) I\left(f / d^{2}\right)
$$

(compare with [Lou2, (5)]).

Lemma 7. We have

$$
I(X)=(\sqrt{X}-1) \log \sqrt{X}+\frac{\kappa_{0}}{2}(\sqrt{X}+1)-J(X)
$$

where

$$
J(X)=\int_{X}^{\infty}(\sqrt{X}+\sqrt{x}) g(x) \frac{d x}{x}=\sqrt{X} \int_{1}^{\infty}(1+\sqrt{x}) g(x X) \frac{d x}{x}
$$

decreases in the range $X \geq 1 /(2 \pi)$ and satisfies

$$
J(X) \leq \frac{2}{\sqrt{X}} \int_{X}^{\infty} g(x) d x=\frac{2}{\pi \sqrt{X}} \sum_{n \geq 1} \frac{1}{n^{2}} e^{-\pi n^{2} X} \leq \frac{2}{\pi \sqrt{X}\left(e^{\pi X}-1\right)} .
$$

Proof. To get (22) and (23), use (15) (see [Lou2]). For $x>0$ the derivative of $X \mapsto \sqrt{X} g(x X)$ which is equal to $\frac{1}{2 \sqrt{X}} \sum_{n \geq 1}\left(1-2 \pi n^{2} x X\right) e^{-\pi n^{2} x X}$ is less than 0 for $X \geq 1 /(2 \pi x)$. Hence, for any $x \geq 1$ the function $X \mapsto$ $\sqrt{X} g(x X)$ decreases in the range $X \geq 1 /(2 \pi)$. Thus, $X \mapsto J(X)$ decreases in the range $X \geq 1 /(2 \pi)$.

Using (21) and (22), we obtain

$$
|L(1, \chi)| \leq \frac{1}{\sqrt{f}} \sum_{d \mid k} \mu(d)\left(\left(\frac{\sqrt{f}}{d}-1\right) \log \left(\frac{\sqrt{f}}{d}\right)+\frac{\kappa_{0}}{2}\left(\frac{\sqrt{f}}{d}+1\right)-J\left(\frac{f}{d^{2}}\right)\right),
$$

which, upon using

$$
\sum_{d \mid k} \mu(d) \frac{\log d}{d}=-\left(\prod_{i=1}^{r}\left(1-\frac{1}{p_{i}}\right)\right) \sum_{i=1}^{r} \frac{\log p_{i}}{p_{i}-1}
$$

yields

$$
|L(1, \chi)| \leq \frac{1}{2}\left\{\prod_{i=1}^{r}\left(1-\frac{1}{p_{i}}\right)\right\}\left(\log f+\kappa_{0}+2 \sum_{i=1}^{r} \frac{\log p_{i}}{p_{i}-1}\right)+\frac{1}{\sqrt{f}} R(f)
$$


with

$$
\begin{aligned}
R(f)= & \Lambda+\varepsilon\left(\kappa_{0}-\log f\right) / 2-\sum_{d \mid k} \mu(d) J\left(f / d^{2}\right) \\
& (\text { and } J \text { as in Lemma } 7), \\
\varepsilon= & \sum_{d \mid k} \mu(d)= \begin{cases}1 & \text { if } r=0, \\
0 & \text { if } r \geq 1,\end{cases} \\
\Lambda= & \sum_{d \mid k} \mu(d) \log d= \begin{cases}-\log p_{1} & \text { if } r=1, \\
0 & \text { if } r \neq 1\end{cases}
\end{aligned}
$$

Now, using (25), we are in a position to complete the proof of Theorem 1.

1. If $r=0$ then $R(f)=\left(\kappa_{0}-\log f\right) / 2-J(f) \leq 0$, and (25) yields $(3)$.

2. If $r=1$ and $p=p_{1}$, then $R(f)=-\log p-J(f)+J\left(f / p^{2}\right) \leq-\log p+$ $J\left(f / p^{2}\right)$, and $f \geq f_{p}:=3 p^{2} /\left(2 \pi^{2}\right)$ implies

$$
R(f) \leq-\log 2+J\left(\frac{3}{2 \pi^{2}}\right) \leq-\log 2+\frac{2 \sqrt{2}}{\sqrt{3}\left(e^{\sqrt{3 / 2}}-1\right)} \leq 0
$$

(by Lemma 7), and (25) yields (4).

3. Assume that $r=2$ and $k=p q$ with $p>q \geq 2$. Set $X=f /\left(p^{2} q^{2}\right)$ and assume that $f \geq f_{p, q}$, i.e. $X \geq(\log 4) /(3 \pi)$. Then

$$
\begin{aligned}
R(f) & =-J(f)+J\left(f / p^{2}\right)+J\left(f / q^{2}\right)-J\left(f /\left(p^{2} q^{2}\right)\right) \\
& \leq J\left(p^{2} X\right)+J\left(q^{2} X\right)-J(X)=\sqrt{X} \int_{1}^{\infty}(1+\sqrt{x}) g_{p, q}(x) \frac{d x}{x}
\end{aligned}
$$

with $g_{p, q}(x)=p g\left(x p^{2} X\right)+q g\left(x q^{2} X\right)-g(x X)$ and $g$ as in (14). We want to prove $R(f) \leq 0$, for (25) would yield (5). It suffices to prove $g_{p, q} \leq 0$ for $x \geq 1$. Now, for $p \geq 2, q \geq 2, n \geq 1, x \geq 1$ and $X \geq(\log 4) /(3 \pi)$ we have

$$
\begin{aligned}
p \exp \left(-\pi n^{2} x\left(p^{2}-1\right)\right. & X)+q \exp \left(-\pi n^{2} x\left(q^{2}-1\right) X\right) \\
& \leq p \exp \left(-\frac{p^{2}-1}{3} \log 4\right)+q \exp \left(-\frac{q^{2}-1}{3} \log 4\right) \\
& \leq 2 \exp (-\log 4)+2 \exp (-\log 4)=1
\end{aligned}
$$

and

$$
p \exp \left(-\pi n^{2} x p^{2} X\right)+q \exp \left(-\pi n^{2} x q^{2} X\right)-\exp \left(-\pi n^{2} x X\right) \leq 0 .
$$

Hence, $g_{p, q}(x) \leq 0$ for $x \geq 1$.

4. Assume that $r \geq 2$ and $f \geq k^{2} /(2 \pi)$. Since there are $2^{r-1}$ divisors $d$ of $k$ for which $\mu(d)<0$ and since $f / d^{2} \geq f / k^{2} \geq 1 /(2 \pi)$ implies 
$0 \leq J\left(f / d^{2}\right) \leq J\left(f / k^{2}\right)$ (use Lemma 7 ), we obtain

$$
R(f)=-\sum_{d \mid k} \mu(d) J\left(f / d^{2}\right) \leq 2^{r-1} J\left(f / k^{2}\right) \leq \frac{2^{r} k}{\pi \sqrt{f}\left(e^{\pi \sqrt{f / k^{2}}}-1\right)}=o(1),
$$

by (24), and (25) yields (2).

\section{Proof of Theorems 4 and 5}

4.1. Notation. Recall that $h=\prod_{i=1}^{s} p_{i}$ and $k=\prod_{i=s+1}^{r} p_{i}$ are two given coprime square-free integers, that $r=s+t$ and that $\chi$ ranges over the primitive even Dirichlet characters of conductors $f=f_{\chi}>1$ such that $k$ divides $f$. We set

$$
\pi_{h}=\prod_{i=1}^{s}\left(1-\frac{1}{p_{i}}\right), \quad \pi_{k}=\prod_{i=s+1}^{r}\left(1-\frac{1}{p_{i}}\right), \quad \pi_{h k}=\pi_{h} \pi_{k}=\prod_{i=1}^{r}\left(1-\frac{1}{p_{i}}\right) .
$$

We also set $\tilde{\pi}_{h}=\prod_{i=1}^{s}\left(1+\frac{1}{p_{i}}\right)$ and

$$
S_{h k}(n):=\sum_{a=1}^{n} \sum_{\substack{b=1 \\ \operatorname{gcd}(b, h k)=1}}^{a} 1 .
$$

Finally, we let $\psi$ be the non-primitive Dirichlet character modulo $F=h f$ induced by $\chi$. Notice that $h k$ divides $F$ and

$$
L(1, \psi)=\left(\prod_{i=1}^{s}\left(1-\frac{\chi\left(p_{i}\right)}{p_{i}}\right)\right) L(1, \chi) .
$$

4.2. Sketch of proofs. So, let $\psi$ be a non-trivial (but not necessarily primitive) even Dirichlet character modulo $F>1$, assume that $h k$ divides $F$ and set

$$
S(n, \psi):=\sum_{a=1}^{n} \sum_{b=1}^{a} \psi(b) .
$$

Since $\psi$ is even and non-trivial, $n \mapsto S(n, \psi)$ is $F$-periodic, hence bounded, and we have the following expression for $L(1, \psi)$ as an absolutely convergent series:

$$
L(1, \psi)=\sum_{n \geq 1} \frac{2 S(n, \psi)}{n(n+1)(n+2)} .
$$

Now, since $\psi(b)=0$ whenever $\operatorname{gcd}(b, h k)>1$ (for $h k$ divides $F$ ), we have $|S(n, \psi)| \leq S_{h k}(n)$ and we will prove in Lemmas 8 and 11 that

$$
|S(n, \psi)| \leq \begin{cases}S_{h k}(n)=\frac{1}{2} n^{2} \pi_{h k}+O(n) & \text { for } n \geq 1 \\ n 2^{s-1} \pi_{k} \sqrt{f}+O(n)+O(\sqrt{f}) & \text { for } 1 \leq n<f / k \\ n 2^{s-1} \sqrt{f}+O(n) & \text { for } n \geq 1\end{cases}
$$


Since the first bound is better than the second for $n<2^{s} \sqrt{f} / \pi_{h}$, and the second is better than the third for $n<f / k$, it is natural to split (28) and write that for any integers $B>A>1$ (in fact, we will also be allowed to choose $B=\infty$ ) we have

$$
|L(1, \psi)| \leq \Sigma_{1}(A, h k)+\Sigma_{2}(A, B, \psi)+\Sigma_{3}(B, \psi)
$$

where

$$
\begin{aligned}
\Sigma_{1}(A, h k) & :=\sum_{n=1}^{A-1} \frac{2 S_{h k}(n)}{n(n+1)(n+2)}, \\
\Sigma_{2}(A, B, \psi) & :=\sum_{n=A}^{B-1} \frac{2|S(n, \psi)|}{n(n+1)(n+2)}, \\
\Sigma_{3}(B, \psi) & :=\sum_{n \geq B} \frac{2|S(n, \psi)|}{n(n+1)(n+2)} .
\end{aligned}
$$

Now, using (29), we will obtain explicit bounds on these $\Sigma_{i}$ in Propositions 9 and 12. Then we will choose $A=\left[2^{s-1} \sqrt{f} / \pi_{k}\right]$ and $B=[f / k]$ to get Theorem 4 . To prove Theorem 5 we will prove in Lemma 11 that the second bound $|S(n, \psi)| \leq n 2^{s-1} \pi_{k} \sqrt{f}+O(n)+O(\sqrt{f})$ is valid for all $n \geq 1$ in the special cases that $t \leq 1$ and we will choose $A=\left[2^{s} \sqrt{f} / \pi_{h}\right]$ and $B=\infty$.

4.3. Bounds on the sum $\Sigma_{1}(A, h k)$

LEmma 8. Let $\{x\} \in[0,1[$ denote the fractional part of a real number $x$ and set

$$
F_{h k}(n):=\sum_{d \mid h k} \mu(d)\left(\sum_{a=1}^{n}\left\{\frac{a}{d}\right\}\right) .
$$

Then

$$
\begin{gathered}
S_{h k}(n)=\pi_{h k} \frac{n(n+1)}{2}-F_{h k}(n), \\
L_{h k}:=\sum_{n \geq 1} \frac{2 F_{h k}(n)}{n(n+1)(n+2)}=\sum_{d \mid h k} \mu(d) \frac{\log d}{d}=-\pi_{h k} \sum_{p \mid h k} \frac{\log p}{p-1},
\end{gathered}
$$

and if $h k>1$, then $n \mapsto G_{h k}(n):=F_{h k}(n)+\pi_{h k} n / 2$ is hk-periodic.

Proof. Since $\sum_{d \mid m} \mu(d)=0$ or 1 according as $m=1$ or $m>1$, we obtain

$$
\begin{aligned}
S_{h k}(n) & =\sum_{a=1}^{n} \sum_{\substack{b=1 \\
\operatorname{gcd}(b, h k)=1}}^{a} 1=\sum_{a=1}^{n} \sum_{b=1}^{a} \sum_{d \mid \operatorname{gcd}(b, h k)} \mu(d)=\sum_{d \mid h k} \mu(d) \sum_{a=1}^{n} \sum_{\substack{b=1 \\
d \mid b}}^{a} 1 \\
& =\sum_{d \mid h k} \mu(d) \sum_{a=1}^{n}\left(\frac{a}{d}-\left\{\frac{a}{d}\right\}\right)=\pi_{h k} \frac{n(n+1)}{2}-F_{h k}(n) .
\end{aligned}
$$


To prove (35), notice that

$$
\begin{aligned}
L_{h k}= & \sum_{n \geq 1} \frac{2}{n(n+1)(n+2)} \sum_{d \mid h k} \mu(d)\left(\sum_{a=1}^{n}\left\{\frac{a}{d}\right\}\right) \\
= & \sum_{d \mid h k} \mu(d) \sum_{a \geq 1}\left\{\frac{a}{d}\right\} \sum_{n \geq a} \frac{2}{n(n+1)(n+2)} \\
= & \sum_{d \mid h k} \mu(d) \sum_{a \geq 1}\left\{\frac{a}{d}\right\}\left(\frac{1}{a}-\frac{1}{a+1}\right) \\
= & \sum_{d \mid h k} \frac{\mu(d)}{d} \sum_{x=1}^{d-1} x \sum_{y \geq 0}\left(\frac{1}{d y+x}-\frac{1}{d y+x+1}\right) \\
& (\text { write } a=d y+x \text { with } 1 \leq x \leq d \text { and } y \geq 0) \\
= & \sum_{d \mid h k} \frac{\mu(d)}{d} \lim _{N \rightarrow \infty} \sum_{x=1}^{d-1} x \sum_{y=0}^{N}\left(\frac{1}{d y+x}-\frac{1}{d y+x+1}\right) \\
= & \sum_{d \mid h k} \frac{\mu(d)}{d} \lim _{N \rightarrow \infty}\left(\left(\sum_{x=1}^{d} \sum_{y=0}^{N} \frac{1}{d y+x}\right)-d \sum_{y=0}^{N} \frac{1}{d y+d}\right) \\
= & \sum_{d \mid h k} \frac{\mu(d)}{d} \lim _{N \rightarrow \infty}\left(\sum_{z=1}^{d(N+1)} \frac{1}{z}-\sum_{z=1}^{N+1} \frac{1}{z}\right)=\sum_{d \mid h k} \frac{\mu(d)}{d} \log d .
\end{aligned}
$$

Finally, assume that $m>n \geq 1$ satisfy $m \equiv n(\bmod h k)$. Then

$$
\begin{aligned}
G_{h k}(m)-G_{h k}(n) & =F_{h k}(m)-F_{h k}(n)+\frac{m-n}{2} \pi_{h k} \\
& =\sum_{d \mid h k} \mu(d)\left(\frac{m-n}{2 d}+\sum_{a=n+1}^{m}\left\{\frac{a}{d}\right\}\right) .
\end{aligned}
$$

Now, $d$ divides $h k$, hence divides $m-n$, and $a \mapsto\{a / d\}$ is $d$-periodic. Hence,

$$
\sum_{a=n+1}^{m}\left\{\frac{a}{d}\right\}=\frac{m-n}{d} \sum_{a=1}^{d}\left\{\frac{a}{d}\right\}=(m-n) \frac{d-1}{2 d}
$$

and, for $h k>1$, we obtain

$$
G_{h k}(m)-G_{h k}(n)=\frac{m-n}{2} \sum_{d \mid h k} \mu(d)=0,
$$

and so $n \mapsto G_{h k}(n)$ is indeed $h k$-periodic. 
Proposition 9. For $A>1$ a rational integer, we have

$$
\Sigma_{1}(A, h k) \leq \pi_{h k}\left(\sum_{p \mid h k} \frac{\log p}{p-1}+\gamma-\frac{3}{2}+\log A+\frac{1}{2 A}\right)+R_{h k}(A)
$$

where

$$
R_{h k}(A)=\sum_{n \geq A} \frac{2 G_{h k}(n)}{n(n+1)(n+2)} .
$$

Moreover, for $h k>1$ we have $R_{h k}(A)=O\left(1 / A^{2}\right)$, and for $h k \in\{2,3,6\}$ we have $G_{h k} \leq 0$ and $R_{h k}(A) \leq 0$.

Proof. We have

$\Sigma_{1}(A, h k)$

$$
\begin{aligned}
& =\sum_{n=1}^{A-1} \frac{2 S_{h k}(n)}{n(n+1)(n+2)} \\
& =\sum_{n=1}^{A-1} \frac{\pi_{h k} n(n+1)-2 F_{h k}(n)}{n(n+1)(n+2)} \quad(\text { by }(34)) \\
& =\pi_{h k}\left(\sum_{n=1}^{A-1} \frac{1}{n+2}\right)-\sum_{n=1}^{A-1} \frac{2 F_{h k}(n)}{n(n+1)(n+2)} \\
& =\pi_{h k}\left(-\frac{3}{2}+\sum_{n=1}^{A+1} \frac{1}{n}\right)-L_{h k}+\sum_{n \geq A} \frac{2 F_{h k}(n)}{n(n+1)(n+2)} \\
& =\pi_{h k}\left(-\frac{3}{2}+\sum_{n=1}^{A+1} \frac{1}{n}\right)+\pi_{h k} \sum_{p \mid h k} \frac{\log p}{p-1}-\frac{\pi_{h k}}{A+1}+\sum_{n \geq A} \frac{2 G_{h k}(n)}{n(n+1)(n+2)}
\end{aligned}
$$

(by $(35)$ )

$$
=\pi_{h k}\left(\sum_{p \mid h k} \frac{\log p}{p-1}-\frac{3}{2}+\sum_{n=1}^{A} \frac{1}{n}\right)+R_{h k}(A),
$$

and the first result follows. The last assertion is easily proved by using the $h k$-periodicity of $G_{h k}$.

\subsection{Bounds on $|S(n, \psi)|$}

4.4.1. On some previous mistakes in the literature. We point out a loophole in the proof of [Hua1, Lemma 4] which asserts that $|S(n, \psi)| \leq \frac{1}{2} n \sqrt{d}$ for certain (not necessarily primitive) Dirichlet quadratic characters modulo 
$d>1$. Indeed, lines 9 and 10 page 733 of the proof of [Hua1, Lemma 4] are

$$
\begin{aligned}
\sum_{a=1}^{A} \sum_{n=1}^{a}\left(\frac{d}{n}\right) & \leq \frac{1}{2} \sum_{r \mid m}\left|\sum_{a=1}^{A} \sum_{n=1}^{[a / r]}\left(\frac{f}{n}\right)\right| \\
& \leq \frac{1}{2} \sum_{r \mid m} r\left|\sum_{b=1}^{[A / r]} \sum_{n=1}^{b}\left(\frac{f}{n}\right)\right| .
\end{aligned}
$$

First, we point out that the factor $\frac{1}{2}$ should be omitted. Second, the only obvious way to warrant the second inequality is to say that for any $r$ we have

$$
\left|\sum_{a=1}^{A} \sum_{n=1}^{[a / r]}\left(\frac{f}{n}\right)\right| \leq r\left|\sum_{b=1}^{[A / r]} \sum_{n=1}^{b}\left(\frac{f}{n}\right)\right|,
$$

which is false. Indeed, choose $f$ such that $\left(\frac{f}{2}\right)=\left(\frac{f}{3}\right)=-1$ (e.g., $f=29$ ), assume that $[A / r]=3$ and write $A=3 r+R_{A}$ with $0 \leq R_{A}<r$. Then

$\sum_{a=1}^{A} \sum_{n=1}^{[a / r]}\left(\frac{f}{n}\right)=\sum_{n=1}^{[A / r]}(A+1-n r)\left(\frac{f}{n}\right)=\sum_{n=1}^{3}(A+1-n r)\left(\frac{f}{n}\right)=r-R_{A}-1$

and

$$
\sum_{b=1}^{[A / r]} \sum_{n=1}^{b}\left(\frac{f}{n}\right)=\sum_{b=1}^{3} \sum_{n=1}^{b}\left(\frac{f}{n}\right)=0
$$

and if $R_{A} \neq r-1$ then (36) is false. For example, (36) is false for $(f, A, r)=$ $(29,9,3)$.

It seems that Hua himself realised that there was a problem with his proof, for in [Hua2, Th. 12.13.2] he gives a correct proof of a similar but slightly worse result according to which $|S(n, \psi)| \leq n \sqrt{d}$ for the same kind of characters. Unfortunaly, Hua's wrong bound $|S(n, \psi)| \leq \frac{1}{2} n \sqrt{d}$ is, for example, used in [SSW, Section 3] (see formulae (3.1)-(3.3) of [SSW]). If one uses the correct bound $|S(n, \psi)| \leq n \sqrt{d}$ then one gets an extra $\frac{1}{2} \log 2$ factor in [SSW, bound (3.7)] and an extra $\frac{1}{3} \log 2$ factor in [SSW, bound (3.11)]. The problem is that the bound [SSW, bound (3.11)] is quoted in [Le, Lemma 2] and used to prove the main result of [Le]. However, as the proof of (8) is correct, the main results of [SSW] and [Le] are correct, even though their proofs stem from a wrong proof of [SSW, bound (3.11)]. Lemma 11 below corrects the statement and proof of [Hua1, Lemma 4] and improves upon [Hua2, Th. 12.13.2].

4.4.2. Bounds on $|S(n, \psi)|$ for primitive even characters. Let us stick to the notation we set in Subsection 4.1. In particular, $t$ denotes the number of prime factors of $k$. 
Lemma 10. Let $\chi$ be a primitive even Dirichlet character modulo $f>1$. Then

$$
2|S(n, \chi)| \leq n \sqrt{f} \quad \text { for } n \geq 1 .
$$

Moreover, if $k$ divides $f$, then

$$
2|S(n, \chi)| \leq \pi_{k}(n+1) \sqrt{f} \quad \text { for } \begin{cases}n \geq 1 & \text { if } t=1 \\ 1 \leq n<f / k & \text { if } t>1 .\end{cases}
$$

Proof. Recall that since $\chi$ is even and non-trivial, $n \mapsto S(n, \chi)$ is $f$-periodic and $S(f, \chi)=0$. Hence, we may assume that $1 \leq n<f$. According to the proofs of [Hua1, Lemma 3] and [Hua2, Theorems 7.9.2 and 12.13.1] (see also the proofs of [Nar, Lemma 8.4] or [MP, Lemme 3.1]), we have

$$
2 \sqrt{f}|S(n, \chi)|=\sqrt{f}\left|\sum_{a=0}^{n} \sum_{b=-a}^{a} \chi(b)\right| \leq \sum_{x=1}^{f}|\chi(x)| \sum_{a=0}^{n} \sum_{b=-a}^{a} e^{2 \pi i b x / f} .
$$

First, since $\chi(f)=0$ and $|\chi(x)| \leq 1$ for $1 \leq x \leq f-1$ and since for $0 \leq|b|<f$ we have

$$
\sum_{x=1}^{f-1} e^{2 \pi i b x / f}= \begin{cases}-1 & \text { if } b \neq 0 \\ f-1 & \text { if } b=0\end{cases}
$$

we obtain $2 \sqrt{f}|S(n, \chi)| \leq f(n+1)-(n+1)^{2}$. Now, $f(n+1)-(n+1)^{2}<n f$ if and only if $n \geq[\sqrt{f}]$, whereas for $0 \leq n \leq[\sqrt{f}]-1$ we also have the trivial bound $2 \sqrt{f}|S(n, \chi)| \leq n(n+1) \sqrt{f} \leq n[\sqrt{f}] \sqrt{f} \leq n f$.

Second, if $k>1$ divides $f$ then $\chi(x)=0$ if $\operatorname{gcd}(x, k)>1$. Hence, using $\sum_{d \mid m} \mu(d)=1$ or 0 according as $m=1$ or $m>1$, we obtain

$$
\begin{aligned}
2 \sqrt{f}|S(n, \chi)| & \leq \sum_{\substack{x=1 \\
\operatorname{gcd}(x, h)=1}}^{f} \sum_{a=0}^{n} \sum_{b=-a}^{a} e^{2 \pi i b x / f} \\
& =\sum_{d \mid h} \mu(d) \sum_{\substack{x=1 \\
d \mid x}}^{f} \sum_{a=0}^{n} \sum_{b=-a}^{a} e^{2 \pi i b x / f} \\
& \leq \sum_{d \mid k} \mu(d) \sum_{x=1}^{f / d} \sum_{a=0}^{n} \sum_{b=-a}^{a} e^{2 \pi i b x /(f / d)} \\
& =\sum_{d \mid k} \mu(d) \sum_{a=0}^{n} \sum_{b=-a}^{a} \frac{f}{d} \\
& =\pi_{k}(n+1) f+2 f \sum_{d \mid k} \frac{\mu(d)}{d} \sum_{a=1}^{n}\left[\frac{a d}{f}\right],
\end{aligned}
$$


which is equal to $\pi_{k}(n+1) f$ for $1 \leq n<f / k$ (for we have $0 \leq a d / f \leq$ $n k / f<1$ ) and is $\leq \pi_{k}(n+1) f$ for $1 \leq n<f$ if $t=1$ (for in that case $k=p$ is prime, $d=1$ or $d=p$, and if $d=1$ then $0 \leq a d / f \leq n d / f=n / f<1$, whereas if $d=p$ we have $\mu(d)=-1 \leq 0)$.

Notice that if $k=6, f=12$ and $n=2$ then

$$
2 f \sum_{d \mid k} \frac{\mu(d)}{d} \sum_{a=1}^{n}\left[\frac{a d}{f}\right]=4>0 .
$$

Hence, our proof does not make it possible to get rid of the restriction $1 \leq n<f / k$ for $t \geq 2$.

4.4.3. Bounds on $|S(n, \psi)|$ for non-primitive even characters. Let us stick to the notation we set in Subsection 4.1. In particular, $s$ denotes the number of prime factors of $h$ and $t$ denotes the number of prime factors of $k$.

Lemma 11. Let $\chi$ be a primitive Dirichlet character of conductor $f$ and let $\psi$ be the non-trivial even Dirichlet character modulo $F=h f$ induced by $\chi$. Then

$$
|S(n, \psi)| \leq n\left(2^{s-1} \sqrt{f}+2^{s}-\widetilde{\pi}_{h}\right),
$$

which yields

$$
|S(n, \psi)|=O(n \sqrt{f}) .
$$

Moreover, if $t=1$ and $n \geq 1$, or if $t \geq 2$ and $1 \leq n<f / k$, then we have the better bound

$$
|S(n, \psi)| \leq n\left(2^{s-1} \pi_{k} \sqrt{f}+2^{s}-\widetilde{\pi}_{h}\right)+\frac{1}{2} \pi_{k} \widetilde{\pi}_{h} h \sqrt{f},
$$

which yields

$$
|S(n, \psi)| \leq n 2^{s-1} \pi_{k} \sqrt{f}+O(n)+O(\sqrt{f}) .
$$

Proof. Since $\psi(b)=\chi(b)$ if $\operatorname{gcd}(b, h)=1$, and $\psi(b)=0$ if $\operatorname{gcd}(b, h)>1$, and since $\sum_{d \mid m} \mu(d)=1$ or 0 according as $m=1$ or $m>1$, we have $\psi(b)=\chi(b) \sum_{d \mid \operatorname{gcd}(b, h)} \mu(d)$ and

$$
\begin{aligned}
S(n, \psi) & =\sum_{a=1}^{n} \sum_{b=1}^{a} \chi(b) \sum_{d \mid \operatorname{gcd}(b, h)} \mu(d) \\
& =\sum_{d \mid h} \mu(d) \sum_{a=1}^{n} \sum_{b=1}^{[a / d]} \chi(d b)=\sum_{d \mid h} \mu(d) \chi(d) \sum_{a=1}^{n} \sum_{b=1}^{[a / d]} \chi(b) .
\end{aligned}
$$


Now, writing $n=d[n / d]+R$ with $0 \leq R<d$ and $a=d x+y$, we obtain

$$
\begin{aligned}
\sum_{a=0}^{n} \sum_{b=0}^{[a / d]} \chi(b) & =\sum_{x=0}^{[n / d]} \sum_{y=0}^{d-1} \sum_{b=0}^{x} \chi(b)-\sum_{y=R+1}^{d-1} \sum_{b=0}^{[n / d]} \chi(b) \\
& =d\left(\sum_{x=0}^{[n / d]} \sum_{b=0}^{x} \chi(b)\right)-(d-R-1) \sum_{b=0}^{[n / d]} \chi(b) \\
& =d S([n / d], \chi)-(d-R-1) \sum_{b=1}^{[n / d]} \chi(b)
\end{aligned}
$$

and

$$
\begin{aligned}
|S(n, \psi)| & \leq \sum_{d \mid h}|\mu(d)|(d|S([n / d], \chi)|+(d-1)[n / d]) \\
& \left.\leq \frac{n}{2} \sum_{d \mid h}|\mu(d)|\left(\sqrt{f}+2 \frac{d-1}{d}\right) \quad \text { (by }(37)\right) \\
& =n\left(2^{s-1} \sqrt{f}+2^{s}-\widetilde{\pi}_{h}\right) .
\end{aligned}
$$

Moreover if we use (38) instead of (37), then for $1 \leq n<f / k$ we have $[n / d] \leq n / d \leq n<f / k$ and

and we obtain

$$
|S([n / d], \chi)| \leq \frac{\pi_{k}(n+d) \sqrt{f}}{2 d}
$$

$$
\begin{aligned}
|S(n, \psi)| & \leq \sum_{d \mid h}|\mu(d)|\left(\frac{\pi_{k}(n+d) \sqrt{f}}{2}+n \frac{d-1}{d}\right) \\
& =n\left(2^{s-1} \pi_{k} \sqrt{f}+2^{s}-\widetilde{\pi}_{h}\right)+\frac{1}{2} \pi_{k} \widetilde{\pi}_{h} h \sqrt{f} .
\end{aligned}
$$

4.5. Bounds on the sums $\Sigma_{2}(A, B, \psi)$ and $\Sigma_{3}(B, \psi)$

Proposition 12. Let $\chi$ be a primitive Dirichlet character of conductor $f$ divisible by the given $k$ and let $\psi$ be the non-trivial even Dirichlet character modulo $F=h f$ induced by $\chi$. Then for $1<A \leq B \leq f / k$ we have

$$
\Sigma_{2}(A, B, \psi):=\sum_{n=A}^{B-1} \frac{2|S(n, \psi)|}{n(n+1)(n+2)} \leq \frac{2^{s} \pi_{k} \sqrt{f}}{A+1}+O\left(\frac{1}{A}\right)+O\left(\frac{\sqrt{f}}{A^{2}}\right)
$$

and

$$
\Sigma_{3}(B, \psi):=\sum_{n \geq B} \frac{2|S(n, \psi)|}{n(n+1)(n+2)}=O\left(\frac{\sqrt{f}}{B}\right) .
$$

Proof. The bound on $\Sigma_{3}(B, \psi)$ is easy and follows from (40) and from $\sum_{n \geq B} 1 /((n+1)(n+2))=1 /(B+1)$. In the same way, to obtain the bound 
for $\Sigma_{2}(A, B, \psi)$ we use $(42), \sum_{n=A}^{B-1} 1 /((n+1)(n+2))=1 /(A+1)-1 /(B+1)$ $\leq 1 /(A+1)$ and $\sum_{n=A}^{B-1} 2 /(n(n+1)(n+2))=1 /(A(A+1))-1 /(B(B+1))$ $\leq 1 /(A(A+1))$.

4.6. Proof of Theorem 4. According to Proposition 9 and to Proposition 12 with $B=[f / k]$, we have

$$
\begin{aligned}
|L(1, \psi)| \leq & \Sigma_{1}(A, h k)+\Sigma_{2}(A, B, \psi)+\Sigma_{3}(B, \psi) \\
\leq & \pi_{h k}\left(\sum_{p \mid h k} \frac{\log p}{p-1}+\gamma-\frac{3}{2}+\log A\right) \\
& +\frac{2^{s} \pi_{k} \sqrt{f}}{A+1}+O\left(\frac{1}{A}+\frac{\sqrt{f}}{A^{2}}+\frac{1}{\sqrt{f}}\right),
\end{aligned}
$$

which for the almost optimal choice $A=\left[2^{s} \sqrt{f} / \pi_{h}\right]$ yields

$$
\left|\prod_{i=1}^{s}\left(1-\frac{\chi\left(p_{i}\right)}{p_{i}}\right)\right||L(1, \chi)|=|L(1, \psi)| \leq \frac{1}{2} \pi_{h k}\left(\log f+\kappa_{h k}\right)+O\left(\frac{1}{\sqrt{f}}\right)
$$

with

$$
\kappa_{h k}=s \log 4+2 \sum_{p \mid h k} \frac{\log p}{p-1}+2 \gamma-1-2 \log \pi_{h}=2 \kappa
$$

(use (27)).

4.7. Proof of Theorem 5. To prove item 1 of Theorem 5 , we choose $h=2$ and $k=1$. Hence, $\pi_{h}=1 / 2, \widetilde{\pi}_{h}=3 / 2, \pi_{h k}=1 / 2$ and $\sum_{p \mid h k} \frac{\log p}{p-1}=\log 2$. According to (39) of Lemma 11 we have $2|S(n, \psi)| \leq n(2 \sqrt{f}+1)$ for any $n \geq 1$. Now, we may choose $B=\infty$ to obtain $\Sigma_{3}(B, \psi)=0$ and

$$
\Sigma_{2}(A, \infty, \psi)=\sum_{n \geq A} \frac{2|S(n, \psi)|}{n(n+1)(n+2)} \leq \sum_{n \geq A} \frac{2 \sqrt{f}+1}{(n+1)(n+2)}=\frac{2 \sqrt{f}+1}{A+1} .
$$

Hence, using (27), (30) and Proposition 9 we obtain

$$
\left|\left(1-\frac{\chi(2)}{2}\right) L(1, \chi)\right|=|L(1, \psi)| \leq \frac{1}{2}\left(\log 2+\gamma-\frac{3}{2}+\log A+\frac{1}{2 A}\right)+\frac{2 \sqrt{f}+1}{A+1},
$$

which for the almost optimal choice $A=[4 \sqrt{f}]$ yields $\log A+1 /(2 A) \leq$ $\log (4 \sqrt{f})+1 /(8 \sqrt{f}),(2 \sqrt{f}+1) /(A+1) \leq(2 \sqrt{f}+1) /(4 \sqrt{f})$,

$$
\left|\left(1-\frac{\chi(2)}{2}\right) L(1, \chi)\right| \leq \frac{1}{4}\left(\log f+6 \log 2+2 \gamma-1+\frac{5}{4 \sqrt{f}}\right)
$$

and the desired results (notice that $f \geq 5$ ).

To prove item 2 of Theorem 5 , we choose $h=3$ and $k=2$. Hence, $s=t=1, \pi_{h}=2 / 3, \widetilde{\pi}_{h}=4 / 3, \pi_{k}=1 / 2, \pi_{h k}=1 / 3$ and $\sum_{p \mid h k} \frac{\log p}{p-1}=\log 2+$ $\frac{1}{2} \log 3$. According to (41) of Lemma 11 we have $2|S(n, \psi)| \leq(n+2) \sqrt{f}+4 n / 3$ 
for any $n \geq 1$. Now, we may choose $B=\infty$ to obtain $\Sigma_{3}(B, \psi)=0$ and

$$
\Sigma_{2}(A, \infty, \psi) \leq \sum_{n \geq A} \frac{(n+2) \sqrt{f}+4 n / 3}{n(n+1)(n+2)}=\frac{\sqrt{f}}{A}+\frac{4 / 3}{A+1} .
$$

Hence, using (27), (30) and Proposition 9 we obtain

$$
\begin{aligned}
& \left|\left(1-\frac{\chi(3)}{3}\right) L(1, \chi)\right| \\
& \quad=|L(1, \psi)| \leq \frac{1}{3}\left(\log 2+\frac{1}{2} \log 3+\gamma-\frac{3}{2}+\log A+\frac{1}{2 A}\right)+\frac{\sqrt{f}}{A}+\frac{4 / 3}{A+1},
\end{aligned}
$$

which for the almost optimal choice $A=[3 \sqrt{f}]$ yields

$$
\left|\left(1-\frac{\chi(3)}{3}\right) L(1, \chi)\right| \leq \frac{1}{6}\left(\log f+\log (108)+2 \gamma-1+\frac{3}{\sqrt{f}}+\frac{2}{3 \sqrt{f}-1}\right)
$$

and the desired result for $f \geq 12$. Finally, if $f<12$ then $\chi$ must be the only primitive even quadratic character of conductor 8 for which we also have

$$
\left|\left(1-\frac{\chi(3)}{3}\right) L(1, \chi)\right|=\left(1+\frac{1}{3}\right) \frac{\log (1+\sqrt{2})}{2} \leq(\log 8+6) / 6 .
$$

5. Applications. Using such explicit bounds on $|L(1, \chi)|$, we can deduce explicit upper bounds on class numbers of real abelian number fields or on the lengths of the periods of the continued fractional expansions of real quadratic irrational numbers (see $[\mathrm{Le}],[\mathrm{MP}]$ ) and $[\mathrm{SSW}]$ ), and lower bounds on relative class numbers of imaginary abelian number fields (see [LYK] where the use of Theorem 5 is of paramount importance to make it possible to solve the exponent two class groups problem for some CM-fields of 2-power degrees).

Let us only give here the following illustration of the use of our results to get (in a special case) a 3 -fold improvement on the lower bound given in [Lou4, Theorem 5]:

Proposition 13. Let $\mathbf{K}$ be an imaginary cyclic quartic field of conductor $f_{\mathbf{K}}$. Let $\mathbf{k}$ denote the real quadratic subfield of $\mathbf{K}$. Assume that the relative class number $h_{\mathbf{K}}^{-}$of $\mathbf{K}$ is odd and that $f_{\mathbf{K}} \neq 16$. Then $f_{\mathbf{K}}=p \equiv 5$ $(\bmod 8)$ is prime, $\mathbf{k}=\mathbb{Q}(\sqrt{p}), 2$ is inert in $\mathbf{k}$ and

$$
h_{\mathbf{K}}^{-} \geq \varepsilon_{p} \frac{2 p}{e \pi^{2}(\log p+5) \log p},
$$

where $\varepsilon_{p}=1-\left(4 \pi e^{1 / 2} / p^{3 / 4}\right)$. In particular, if $h_{\mathbf{K}}^{-}=1$ then $p=f_{\mathbf{K}}<1300$.

Proof. Adapt the proof of [Lou4, Theorem 5], but instead of using [Lou4, (9)] which gives $\operatorname{Res}_{s=1}\left(\zeta_{\mathbf{k}}\right)=L\left(1, \chi_{\mathbf{k}}\right) \leq(\log p+0.05) / 2$, use (9) of our present Theorem 5 to obtain $\operatorname{Res}_{s=1}\left(\zeta_{\mathbf{k}}\right)=L\left(1, \chi_{\mathbf{k}}\right) \leq(\log p+5) / 6$. 


\section{References}

[Hua1] L.-K. Hua, On the least solution of Pell's equation, Bull. Amer. Math. Soc. 48 (1942), 731-735.

[Hua2] -, Introduction to Number Theory, Springer, 1982.

[Le] M.-H. Le, Upper bounds for class numbers of real quadratic fields, Acta Arith. 68 (1994), 141-144.

[Lou1] S. Louboutin, Majoration au point 1 des fonctions $L$ associées aux caractères de Dirichlet primitifs, ou au caractère d'une extension quadratique d'un corps quadratique imaginaire principal, J. Reine Angew. Math. 419 (1991), 213-219.

[Lou2] -, Majorations explicites de $|L(1, \chi)|$, C. R. Acad. Sci. Paris 316 (1993), 11-14.

[Lou3] -, Majorations explicites de $|L(1, \chi)|$ (suite), ibid. 323 (1996), 443-446.

[Lou4] -, CM-fields with cyclic ideal class groups of 2-power orders, J. Number Theory 67 (1997), 1-10.

[Lou5] -, Majorations explicites de $|L(1, \chi)|$ (troisième partie), C. R. Acad. Sci. Paris 332 (2001), 95-98.

[LYK] S. Louboutin, Y.-S. Yang and S.-H. Kwon, The non-normal quartic CM-fields and the dihedral octic CM-fields with ideal class groups of exponent $\leq 2$, preprint, Univ. Caen, November 1999.

[MP] C. Moser et J.-J. Payan, Majoration du nombre de classes d'un corps cubique de conducteur premier, J. Math. Soc. Japan 33 (1981), 701-706.

[Nar] W. Narkiewicz, Elementary and Analytic Theory of Algebraic Numbers, 2nd ed., Springer, 1990.

[Pin] J. Pintz, Elementary methods in the theory of L-functions VII. Upper bound for $L(1, \chi)$, Acta Arith. 32 (1977), 397-406.

[Ram] O. Ramaré, Approximate formulae for $L(1, \chi)$, ibid. 100 (2001), 245-266.

[SSW] R. G. Stanton, C. Sudler and H. C. Williams, An upper bound for the period of the simple continued fraction for $\sqrt{D}$, Pacific J. Math. 67 (1976), 525-536.

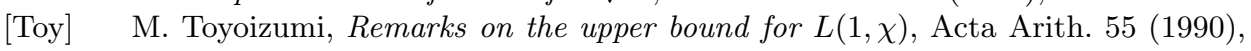
$137-140$.

Institut de Mathématiques de Luminy

UPR 9016

163, avenue de Luminy

Case 907

13288 Marseille Cedex 9, France

E-mail: loubouti@iml.univ-mrs.fr

Received on 11.4.2000

and in revised form on 19.2.2001 